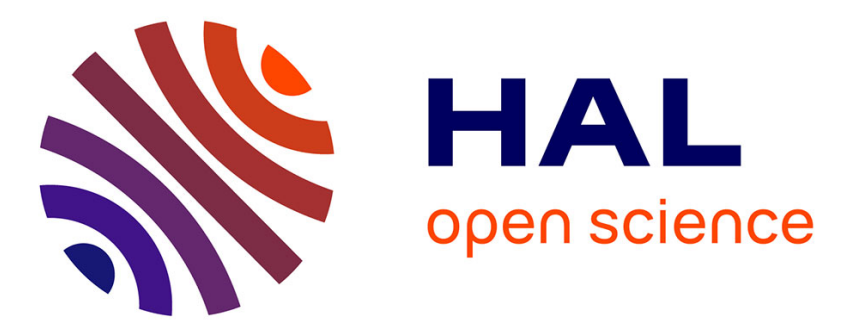

\title{
TRANSFORMATION D'UNE ÉQUATION D'ONDE NON LINÉAIRE EN UNE FORME LINÉAIRE APPROCHÉE ANALYTIQUEMENT SOLUBLE
}

\author{
R. Burvingt
}

\section{- To cite this version:}

R. Burvingt. TRANSFORMATION D'UNE ÉQUATION D'ONDE NON LINÉAIRE EN UNE FORME LINÉAIRE APPROCHÉE ANALYTIQUEMENT SOLUBLE. Journal de Physique IV Proceedings, 1992, 02 (C1), pp.C1-725-C1-728. 10.1051/jp4:19921157 • jpa-00251116

HAL Id: jpa-00251116 https://hal.science/jpa-00251116

Submitted on 1 Jan 1992

HAL is a multi-disciplinary open access archive for the deposit and dissemination of scientific research documents, whether they are published or not. The documents may come from teaching and research institutions in France or abroad, or from public or private research centers.
L'archive ouverte pluridisciplinaire HAL, est destinée au dépôt et à la diffusion de documents scientifiques de niveau recherche, publiés ou non, émanant des établissements d'enseignement et de recherche français ou étrangers, des laboratoires publics ou privés. 


\title{
TRANSFORMATION D'UNE EQUATION D'ONDE NON LINEAIRE EN UNE FORME LINEAIRE APPROCHÉE ANALYTIQUEMENT SOLUBLE
}

\author{
R. BURVINGT \\ Le Centre Thomson d'Applications Radars, 6 rue Nieuport, F-78143 Vélizy-Villacoublay cedex, France
}

\begin{abstract}
Résumé
On exprime une équation non linéaire relative à des ondes approximativement progressives et du troisième ordre par rapport au champ. Les mouvements fluides sont supposés irrotationnels, le milieu peut être inhomogène et la dissipation est considérée. Pour cette équation il est présenté une méthode de résolution ne faisant pas intervenir d'échelles multiples. On utilise une transformation intégrale qui permet de réécrire l'équation initiale sous la forme d'une équation linéaire approchée. Dans cette dernière des dérivées partielles rendent approximativement compte des non-linéarités. Elle peut être mise sous la forme d'une équation de HELMHOLTZ.
\end{abstract}

\begin{abstract}
$\underline{\text { Abstract }}$
A third order nonlinear equation for almost progressive waves is formulated. Dissipation and inhomogenous media are considered. The movements are irrotational. For the equation we present a method of resolution which does not take into account multiple scales. We use an integral transform allowing the derivation from the initial equation of an approached linear equation. In this new equation partial derivatives approximate the nonlinearities. It can be expressed as a HELMHOLTZ equation.
\end{abstract}

\section{INTRODUCTION}

Lorsque, dans un fluide, les puissances mises en jeu pour produire le son deviennent importantes, des effets non linéaires, apparaissent induisant la production d'harmoniques.

Si les amplitudes alors finies du son ne sont pas trop grandes (les effets de cavitation ne se produisent pas), les effets non linéaires peuvent être pris en compte dans les équations en retenant certains termes que l'on peut estimer petits. Bon nombre d'auteurs ont développé des méthodes de descriptions de ces effets [1-3,...]. Ces méthodes font intervenir le plus souvent les échelles multiples et on prend alors en compte des équations où l'approximation du second ordre par rapport à l'amplitude du champ est considérée.

L'étude exposée ici porte sur ces effets non linéaires et l'on retient l'hypothèse d'ondes approximativement progressives. La majeure partie des résultats obtenus dans [4], relatifs à des millieux homogènes, sont évoqués et, on présente une extension de ceux-ci aux milieux inhomogènes. On écrit alors une équation du troisième ordre par rapport à l'amplitude du champ déduite des équations de la mécanique des fluides. Les termes dépendant de la dissipation y sont supposés être du second et du troisième ordre, et on fait l'hypothèse de mouvements irrotationnels. 
Dans les développements qui suivent, une forme simplifiée de cette équation est considérée où seul le terme dominant de, l'ensemble des termes rendant compte de la dissipation, est retenu. On propose une méthode de résolution basée sur la transformation de l'équation non-linéaire du potentiel des vitesses en une forme linéaire approchée. Cette forme peut s'exprimer comme une équation de HELMHOLTZ. Pour le cas d'une source plane directive et d'un milieu homogène, le domaine de validité de la solution approchée obtenue à partir de cette forme est estimé.

\section{EOUATION D'ONDES}

En un point d'observation $\mathrm{O}^{\prime}$ dans un fluide, on considère un repère local $\left(\mathrm{O}^{\prime}, \mathrm{n}_{\mathrm{o}}, \mathrm{n}_{1}, \mathrm{n}_{2}\right)$. Dans l'hypothèse $\mathrm{d}^{\prime}$ onde approximativement progressives suivant l'axe $\overrightarrow{O^{\prime} n_{0}}$, le potentiel des vitesses $\varphi$ peut être écrit sous la forme :

$$
\varphi=\varphi\left(t, \mathrm{n}_{0}, \sqrt{\varepsilon_{\mathrm{d}}} \mathrm{n}_{1}, \sqrt{\varepsilon_{\mathrm{d}}} \mathrm{n}_{2}\right) \text {, avec } \varepsilon_{\mathrm{d}}<1
$$

Le paramètre $\varepsilon_{d}$ rend compte de la lenteur des variations de $\varphi$ suivant $\overrightarrow{O^{\prime} n_{i}}(i=1,2)$ devant celle suivant $\overrightarrow{O^{\prime} n_{0}}$.

Dans [4] une équation non linéaire du troisième ordre par rapport à $\varphi$ est exprimée pour un milieu homogène, et les développements qui y sont retenus peuvent être étendus à la considération d'un milieu inhomogène. Il vient l'équation :

$$
\begin{gathered}
\varphi_{\mathrm{tt}}-\mathrm{c}^{2} \Delta \varphi+\beta \frac{\partial}{\partial \mathrm{t}}\left(\left(\varphi_{\mathrm{t}}^{2}\right)+\zeta \frac{\partial}{\partial \mathrm{t}}\left(\varphi_{\mathrm{t}}^{3}\right)=\right. \\
\mu \varphi_{\mathrm{ttt}}+\mu^{\prime} \varphi_{\mathrm{tttt}}+\mu^{\prime \prime} \frac{\partial^{2}}{\partial \mathrm{t}^{2}}\left(\varphi_{\mathrm{t}}^{2}\right)+\mu^{\prime \prime \prime} \varphi_{\mathrm{tt}}^{2}+\alpha\left(\beta \varepsilon_{\mathrm{d}}\right),
\end{gathered}
$$

où le potentiel $\varphi$

est exprimé sous forme adimensionelle $(\varphi=\mathrm{O}(1)), c, \beta, \zeta, \mu, \mu^{\prime}, \mu^{\prime \prime}, \mu^{\prime \prime \prime}$ sont fonction s de $\mathrm{O}^{\prime}$. Le coefficient $\beta$ est de l'ordre de grandeur d'un nombre de $\mathrm{MACH}$ de référence et, $\zeta=\mathrm{O}\left(\beta^{2}\right)$, et $\mu^{\prime}, \mu^{\prime \prime}, \mu^{\prime \prime \prime}$, sont de l'ordre de $\beta \mu$. Si le milieu est homogène on a $c=1$.

Dans les calculs présentés plus loin on considère que $\mu^{\prime}=\mu^{\prime \prime}=\mu^{\prime \prime \prime}=0$ et que $O\left(\beta \varepsilon_{\mathrm{d}}\right)=0$.

On retient donc l'équation déduite de (1) :

$$
\varphi_{\mathrm{tt}}-\mathrm{c}^{2} \Delta \varphi+\beta \frac{\partial}{\partial \mathrm{t}}\left(\varphi_{\mathrm{t}}^{2}\right)+\zeta \frac{\partial}{\partial \mathrm{t}}\left(\varphi_{\mathrm{t}}^{3}\right)=\mu \varphi_{\mathrm{ttt}}
$$

Celle-ci est justifiée dans les deux cas suivants : 1) $\mu \ll \beta$, le nombre de GOL'DBERG est grand devant un et $\varepsilon<<\quad \beta$ (la divergence du faisceau d'ondes est faible) ; 2) $\zeta$ est négligeable, on est dans le cadre de l'approximation où seul le second ordre est retenu [5]. Il n'existe alors pas de restriction sur le nombre de GOL'BERG et on retient $\varepsilon_{\mathrm{d}} \ll 1$ (on peut avoir $\varepsilon_{\mathrm{d}} \sim 0(\beta)$ et la divergence du faisceau peut être plus importante que dans le cas précédent). 


\section{REDUCTION DE L'EOUATION NON LINEAIRE A UNE FORME LINEAIRE APPROCHEE}

On considère la fonctionnelle non linéaire de $\varphi$ suivant :

$$
F_{1}=\frac{1}{-i b} \int_{0}^{\infty} e^{-p t}\left(e^{-i b p t}-1\right) d t \quad \text { où }: \text { Réel }(p)>0 \text { et } b \in R
$$

On retient de plus qu'à l'instant initial $t=0: \varphi_{t}=\varphi_{t t}=\varphi_{t t t}=0$. Des intégrations par partie conduisent à déduire de (2) l'équation :

$$
\begin{gathered}
\left(\mathrm{p}^{2}-\mu \mathrm{p}^{3}\right) \mathrm{F}_{1}-\mathrm{c}^{2} \Delta F_{1}+2 \mathrm{i} \beta \mathrm{p}^{2} \frac{\partial \mathrm{F}_{1}}{\partial \mathrm{b}}-3 \zeta \mathrm{p}^{2} \frac{\partial^{2} \mathrm{~F}_{1}}{\partial \mathrm{b}^{2}}=\mathrm{ibH} \\
\text { où : } \quad \mathrm{H}=\int_{0}^{\infty} \mathrm{e}^{-\mathrm{pt}-\mathrm{ib} \varphi_{1}\left(\mathrm{~h}^{(\zeta)}+\mathrm{h}^{(\mu)}+\mathrm{h}^{(\mathrm{d})}\right) \mathrm{dt}}
\end{gathered}
$$

avec : $h^{(\mu)}=\mu p \varphi_{t t}^{2}$ et en première approximation :

$$
h^{(\zeta)}=\beta^{2} \varphi_{\mathfrak{t}}^{2} \varphi_{\mathfrak{t t}}^{2} ; h^{(d)}=\left(\frac{\partial \varphi_{t}}{\partial n_{1}}\right)^{2}+\left(\frac{\partial \varphi_{t}}{\partial n_{2}}\right)^{2}-\varphi_{\mathfrak{t t}}\left(\frac{\partial^{2} \varphi}{\partial n_{1}^{2}}+\frac{\partial^{2} \varphi}{\partial n_{2}^{2}}\right)=\alpha\left(\varepsilon_{d}\right)
$$

On remarque que dans $\mathrm{H}$ ne figure pas de terme de l'ordre de $\beta$. Le potentiel $\varphi$ s'obtient à partir de $\mathrm{F}_{1}$ à l'aide de la relation

$$
\mathrm{p} L(\varphi)=\mathrm{L}\left(\varphi_{\mathrm{t}}\right)=\mathrm{F}_{1}(\mathrm{~b}=0)
$$

où le symbole $L$ signifie une application de la transformation de LAPLACE sur $t(t \rightarrow p)$.

Si le second membre de l'équation (3) est négligé $(\mathrm{H} \sim 0)$, après transformation de FOURIER sur $b$, on obtient à partir de celle-ci l'équation de type HELMHOLTZ :

$$
\begin{gathered}
\Delta F_{2}-\frac{p^{2}}{c^{2}}\left(1+2 \beta \lambda+3 \zeta \lambda^{2}-\mu p\right) F_{2}=0 \\
\text { où : } \quad F_{2}=(T F)_{b \rightarrow \lambda} F_{1} .
\end{gathered}
$$

si une résolution de cette dernière équation (linéaire) est développée, on peut exprimer une approximation de $\varphi$ telle que :

$$
L(\varphi)=\frac{1}{p} \int_{-\infty}^{+\infty} F_{2} d \lambda
$$

Une évaluation du domaine de validité de (6) n'a pas été effectuée. Cependant des calculs relatifs à des milieux homogènes $(c=1)$ paraissent indiquer qu'il peut être grand.

\section{CONSIDERATION SUR L'APPLICATION DE LA METHODE AU RAYONNEMENT NON LINEAIRE D'UNE SOURCE PLANE DANS UN MILIEU HOMOGENE}

Pour une source plane et un milieu homogène, on obtient dans [4] une expression approchée du potentiel formulée dans un espace symbolique. Cet espace est celui qui est à retenir après : (i) une transformation de LAPLACE par rapport à la coordonnée normale à la source et (ii) une transformation de FOURIER dans le plan de cette dernière.

Si le régime est stationnaire, et si dans les non-linéarités de (2), seule celle du second ordre est retenue $(\zeta=0)$, la transformation de LAPLACE inverse (relative à la coordonnée normale) peut être aisément opérée. 
On obtient alors [4] une expression de la solution approchée et celle d'une correction donnée par la prise en compte $\mathrm{du}$ second membre de (2). Ces deux expressions sont propices à une analyse physique. La correction due à $h(d)$ (4) apparaît comme faiblement séculaire en fonction de la coordonnée normale.

Dans le cas d'une source gaussienne et si $\mu<\beta$, on peut estimer que cette solution est justifiée dans un domaine de l'espace fluide situé entre la source et le lieu des points où existerait une onde de choc dans le cas non dissipatif $(\mu=0)$. On peut prévoir qu'elle reste justifiée pour des ondes se propageant sur une certaine distance au-delà de ce lieu. On note que dans le cas d'ondes unidimensionelles et d'une source harmonique, elle conduit à la solution de FUBINI [6] lorsque la dissipation est supposée nulle $(\mu=0)$.

\section{CONCLUSION}

Une équation du troisième ordre par rapport à l'amplitude du champ est écrite dans le cas d'ondes approximativement progressives. Elle peut être transformée en une forme linéaire approchée pour laquelle des méthodes de résolution existent. Des calculs plus justes, ne retenant pas cette seul forme linéaire, pourraient être développés en prenant en compte un terme correctif (fonctionnelle non linéaire). Pour cela une méthode de résolution par approximations successives pourrait être retenue.

La méthode exposée devrait permettre par exemple une approche nouvelle dans l'étude des antennes paramétriques et dans celle des effets non linéaires pouvant exister dans les chenaux acoustiques.

\section{BIBLIOGRAPHIE}

[1] J.N. TJOTTA, F. TJOTTA, J.AS.A. N69, pp. 1644-1652 (1981).

[2] F. COULOUVRAT, Thèse de l'Université PARIS 6 (septembre 1991).

[3] T. TANUITI, Wave Motion 12, pp. 373-383 (1990).

[4] R. BURVINGT, article à paraître dans le Journal d'Acoustique.

[5] V.P. KUZNETSOV, S.P.A., Vd 16, n 4, pp. $467-470$ (1971).

[6] B. POIREE, D. ODERO, revue du CETHEDEC, n $46,1976$. 